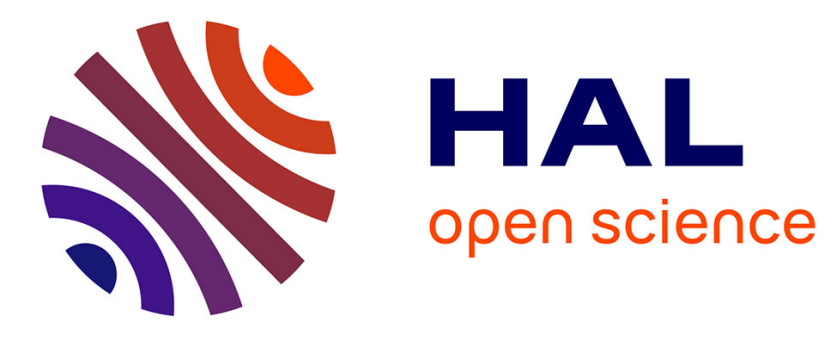

\title{
Trajectoires militantes et reconversions
}

Françoise Blum

\section{To cite this version:}

Françoise Blum. Trajectoires militantes et reconversions : à propos de la Fédération des étudiants d'Afrique noire en France (FEANF) et des années 68. Genèses. Sciences sociales et histoire, 2017, 107, pp.106-130. halshs-01546837

\section{HAL Id: halshs-01546837 https://shs.hal.science/halshs-01546837}

Submitted on 26 Jun 2017

HAL is a multi-disciplinary open access archive for the deposit and dissemination of scientific research documents, whether they are published or not. The documents may come from teaching and research institutions in France or abroad, or from public or private research centers.
L'archive ouverte pluridisciplinaire HAL, est destinée au dépôt et à la diffusion de documents scientifiques de niveau recherche, publiés ou non, émanant des établissements d'enseignement et de recherche français ou étrangers, des laboratoires publics ou privés. 
Trajectoires militantes et reconversions : à propos de la Fédération des étudiants d'Afrique noire en France (FEANF) et des années 68.

\section{Françoise Blum}

«La FEANF l'avait dopé [Lazare Matsocota]. Elle avait donné du corps aux ambitions politiques qu'il nourrissait depuis toujours. La parcelle de pouvoir qu'il avait endossée à l'occasion de ses missions faisait son œuvre. Le pouvoir ? II en raffolait. Comme beaucoup d'étudiants africains dont la FEANF avait libéré et conforté les ambitions, il s'y voyait et n'en démordait plus. Mais contrairement à la plupart de ses aspirants dirigeants politiques qui confondaient supériorité intellectuelle et talent politique, il ne se contentait pas d'en rêver et ne se fiait pas qu'à ses seules dispositions naturelles. Il s'y préparait...» (Gnali 2001 : 33)

Quelques études, émanant le plus généralement d'anciens témoins, ont abordé le militantisme étudiant africain en diaspora, à travers I'histoire de l'organisation qui en fut l'instance la plus représentative, la FEANF (Fédération des étudiants d'Afrique noire en France). Les anciens présidents ou membres de son comité exécutif Amady Aly Dieng (2003 2009), Sékou Traoré (1985) ou Charles Diané (1971) ont ainsi raconté "leur" histoire, plus ou moins documentée, de ce qui fut statutairement une association loi de 1901, déclarée le 8 février 1951, et fonctionna tout à la fois comme une association d'entraide, un syndicat, un parti voire un mouvement social. Mais ces monographies, aussi talentueuses soient-elles, et l'on pense particulièrement à Amady Aly Dieng récemment disparu ${ }^{1}$ [fig.1], ont ignoré, parce que leurs auteurs, historiens et témoins, ne l'ont pas vécue, l'histoire de la FEANF après les indépendances. Or la FEANF continue à exister après 1960, ses dirigeants à militer, à s'engager, non plus (ou plus simplement) contre le colonialisme, mais aussi le néo-colonialisme manifesté par l'État français et les nouvelles élites africaines au pouvoir. Et ils s'inscrivent alors dans une mouvance radicale, proche du marxisme, du tiers-mondisme...ll s'agira dans cet article ${ }^{2}$ de se pencher sur l'histoire des acteurs qui dirigèrent la fédération et lui donnèrent ses principales orientations. Etre à la FEANF pouvait signifier des attitudes fort différentes : cotiser via les associations nationales ou académiques qui en étaient adhérentes, participer à des « Nuits de l'Afrique » organisées par la fédération, adhérer à des manifestations diverses plus ou moins militantes, siéger dans des instances d'attribution des bourses et logements ${ }^{3}$ etc. Le noyau de l’organisation en était le Comité exécutif - ou bureau - élu par le Congrès annuel. Le dénominateur commun des membres du CE était une extrême politisation, un radicalisme qu'ils ou - très minoritairement - elles, adoucissaient parfois pour ne pas effrayer "la masse " des adhérents. Masse est sans doute un terme trop fort si l'on songe qu'en 1960 il n'y avait encore que 8000 étudiants africains en France. Mais il devient pertinent si l'on considère le taux d'adhésion : En 1952, 1300 étudiants étaient déjà membres de la FEANF (Guimont 1985 : 108). Ils sont 2500 en 1962. Les fluctuations répondent, en effet de miroir, à celles de la vie politique africaine. Néanmoins les chiffres sont généralement à la baisse après les indépendances, corollaire probable d'une sur- 
politisation, et de polémiques idéologiques qui ne mobilisaient pas forcément la base. II y a encore 2500 adhérents en 1971 mais seulement 1500 en $1972^{4}$.

206 individus ont été membres du CE, de 1951 à 1976, date après laquelle la FEANF se dissout ellemême, littéralement, dans les querelles politico-nationalistes, avant même de l'être officiellement par le gouvernement de Raymond Barre le 5 mai 1980. C'est en fait la fin logique d'une association anti-impériale qui a fait son temps. Les enjeux ne sont plus les mêmes et la dissolution vient en quelque sorte entériner la caducité de la fédération, de même qu'elle sanctionne la fin des "privilèges » impériaux ${ }^{5}$. La tendance est, du milieu des années 50 à celui des années 70 , à la radicalisation. Il y a radicalisation d'un discours qui remet en cause l'ordre mondial avant comme après les indépendances : l'ordre mondial bien plutôt que l'ordre social d'ailleurs, à l'exception d'un intérêt tout théorique pour les « masses ». On refuse les compromis symbolisés par les négociations diverses qui ont conduit à l'indépendance. On prône la violence. On fait la part belle aux marxismes, cohabitant, parfois en tension, avec une sensibilité « catholique sociale» à dominante tiers-mondiste. C'est aussi une radicalisation - ou tout au moins transformation des pratiques militantes qui empruntent, sans surprise d'ailleurs, à l'environnement plus global des années 60 , du communisme orthodoxe aux formes multiples de "gauchismes ». II s'agit de militantisme en diaspora, et de militantisme de jeunesse, c'est-à-dire d'un moment où les individus sont, d'une certaine façon, en apesanteur (Mauger 1994) : libres car loin des contraintes familiales et/ou du milieu habituel et libres car ayant devant eux l'avenir de tous les possibles. Les longues années 60 sont celles de l'optimisme où chantent les lendemains : pour les étudiants africains, ce sont ceux des indépendances, d'une Afrique libérée et pour beaucoup d'une Afrique socialiste. Les premières déceptions, devant les réalités politiques des indépendances, ne sont pas encore des désillusions. II n'est pas trop tard pour envisager des changements et partant des libérations plus prometteuses. Les désillusions ne viendront qu'avec les années 80 .

Considérer la FEANF permet aussi de remettre en cause les chronologies usuelles. 1960, l'année des indépendances, est sans conteste une date symboliquement essentielle. Mais, de la fin de la seconde guerre mondiale au milieu des années soixante-dix, les continuités l'emportent sur les ruptures. La FEANF est un acteur, parmi d'autres, des processus de radicalisation à l'œuvre qui se concrétiseront dans les "explosions" des années 68. Elle est un observatoire à partir duquel on peut mieux comprendre ces années 68 en Afrique, riches de mouvements sociaux, avant et après le Mai français. Ses dirigeants sont à l'écoute des évènements de la planète, qu'il s'agisse des guerres de décolonisation en Afrique portugaise ou des luttes anti-apartheid, des interventions de Che Guevara en Afrique ou en Amérique du Sud, des luttes contre la guerre du Vietnam ${ }^{6}$, même si le devenir de I'Afrique reste leur priorité absolue. En d'autres termes, l'histoire de la FEANF, dont les membres 
sont autant de passeurs entre la France et l'Afrique, participe de celle de la globalisation des mouvements sociaux et de la réinsertion du mai français dans le monde global de la contestation. Elle concerne tant l'histoire de la France, que celle de l'Afrique et du monde.

Notre propos aura deux temps. On essaiera en premier lieu de retracer les grandes lignes des transformations discursives et de pratiques initiées par les leaders de la FEANF dans la France des années 50 jusqu'au milieu des années 70. Les décisions et manifestations diverses sont alors collectives, définies en fonction d'un consensus, qui se forge parfois péniblement, mais qui rend impossible d'attribuer plus à l'un qu'à l'autre les événements ou déclarations militantes. Et ce d'autant plus que l'exil et l'ennemi commun, les pouvoirs coloniaux ou "néo-coloniaux ", soudent les rangs. En un second temps, on considérera plusieurs trajectoires ultérieures. Comment reconvertit-on les capitaux militants - et académiques - acquis durant la jeunesse ? On voit alors se distinguer les trajectoires: la destinée des colonisés se disperse dans les destinées plurielles des anciens militant-e-s.

\section{PRATIQUES MILITANTES D'APRES-INDEPENDANCES}

Après 1960 et les indépendances de quatorze colonies françaises d'Afrique sub-saharienne, la FEANF perd donc ce qui en faisait l'alpha et l'omega, le combat anti-colonial. Mais elle retrouve un nouveau souffle pour dénoncer le néo-colonialisme et les liens de domination perdurant entre la France et ses anciennes colonies. Les regards de ses dirigeants se tournent alors vers les nouveaux régimes africains, pour les dénoncer, l'hostilité militante à l'égard de la métropole ne subsistant qu'à titre plus marginal. Ce changement d'objet - tout relatif, un peu comme si les militants de la FEANF ne savaient pas la réinventer -, va de pair avec un changement de stratégie.

Avant 1960, le militantisme de la FEANF s'exprime de façons diverses. Le comité exécutif appelle à des manifestations de rue ou les organise. Le Ministère de la France d'Outre-mer fait régulièrement l'objet de démonstrations hostiles. Dans l'Etudiant d'Afrique noire, toutes les violences du colonialisme sont dénoncées ${ }^{7}$. Les formes prises par le processus qui mènera à la décolonisation, I'Union française, la Loi-cadre dite Loi Defferre, la participation des Africains aux diverses assemblées de la République sont également condamnées comme autant de compromissions honteuses. Outre ces formes discursives d'opposition militante, la FEANF développe toute une gamme de pratiques contestatrices, "négatives » - en opposition - ou " positives » - d'affirmation identitaire -, qui en font aussi le succès et la reconnaissance sur l'échiquier contestataire.

On a déjà évoqué, en matière de pratiques négatives, les manifestations. La grève aussi s'impose. On peut citer, entre autre, les grèves de loyers à la cité universitaire du boulevard Jourdan. Charles 
Diané en mène une, très longue, en 1959, qui d'ailleurs est un échec (Diané 1971 : 157-159). La Maison de la France d'Outre-mer du boulevard Jourdan est un des hauts-lieux de la FEANF à Paris. Réunions et conférences plus ou moins politiques s'y tiennent, à l'appel de la fédération, comme elles se tiennent aussi au 69 boulevard Poniatowski, la Maison des États d'Afrique de l'Ouest. Les deux dernières manifestations de rue auxquelles appelle la FEANF sont celles du 11 février 1960 contre l'explosion de la Bombe A et du 15 février 1961 en réaction à l'assassinat de Lumumba. Mais elles sont suivies d'expulsions nombreuses d'étudiants hors du territoire français (Guimont 1985 ; Blum 2016). Cela amène les dirigeants de la FEANF à deux prises de position : d'une part l'interdiction aux membres de manifester sur le territoire français et, d'autre part, un mot d'ordre de boycott des études en France, rendu possible par l'offre de bourses venant d'Europe de l'Est ${ }^{8}$. II ne sera guère suivi. Toujours est-il qu'après 60 - ou plus exactement après 62 , si l'on prend en compte un temps de doute et incertitude -, la FEANF redéfinit mots d'ordres et pratiques. On s'y oppose désormais au néo-colonialisme, dans une continuité assumée avec les années coloniales et l'on cible les nouveaux États africains. Il y a bien quelques exceptions, avec par exemple le regain d'hostilité à l'égard de l'ancienne métropole lorsque les Français interviennent pour sauver Léon Mba dans le Gabon de 1964 (Blum 2016 : 92-93). Mais ces prises de position et manifestations hostiles à l'État français entraînant de nouvelles expulsions, la FEANF se cantonne de plus en plus à l'opposition aux nouveaux États "néo-coloniaux» africains, et à leurs chefs "valets de l'impérialisme ». Les manifestations se limitent désormais aux "territoires africains" en France : lieux de vie et ambassades. La Maison de la France d'Outre-mer, rebaptisée après 1960 Maison de l'Afrique, est un de ces lieux de vie. Les étudiants font des grèves de loyers, occupent les locaux avec, ou non, séquestration du directeur ou du personnel (Blum 2017). Les résidents obtiennent en 1968 la nomination d'un directeur africain qu'ils ne vont d'ailleurs pas tarder à traiter, aussi, de néocolonialiste. Une longue période d'occupations-séquestrations, pour divers motifs, se poursuit jusqu'en 1976. La Fondation nationale de la Cité universitaire reprend alors en main le pavillon, nomme un nouveau directeur et dépossède habilement les ambassades - qui ne se privaient pas de défendre leurs étudiants lors même qu'elles s'opposaient à eux dans d'autres arènes - de tout pouvoir sur le pavillon.

Outre ce lieu de vie, les occupations ont pour terrain privilégié les diverses ambassades africaines. Le scénario est toujours le même : occupation de l'ambassade par un groupe multi-national d'étudiants (les ressortissants de l'ambassade occupée sont généralement minoritaires), séquestration éventuelle de l'ambassadeur, intervention des forces de police à la demande de l'ambassade, libération des locaux, interpellations, relaxes ${ }^{9}$. Ces pratiques d'occupation sont alors les mêmes que celles qui prévalent, en amont et en aval de Mai 68 sur l'ensemble du territoire français, qu'il s'agisse 
d'occupations d'usines, de lycées ou d'universités. Les étudiants africains empruntent au répertoire d'action en vigueur leurs pratiques de contestation de leurs gouvernants. Ils sauront, aussi, utiliser ce répertoire en Afrique même, si l'on songe par exemple aux occupations des universités de Dakar en mai 1968, et de Tananarive, en mai 1972 (Blum 2014). Néanmoins, la FEANF « manque » quelque peu Mai 68 , ce qui sera d'ailleurs reproché au bureau lors du congrès de décembre 68 , même si des étudiants africains y participent individuellement - le plus célèbre exemple étant celui d'Omar Blondin Diop, le héros maoïste de la Chinoise ${ }^{10}$ qui prend part aux évènements du 22 mars, et sera quelques années plus tard assassiné dans la prison de Gorée. La fédération manifeste néanmoins sa solidarité et se rapproche de l'extrême-gauche française.

D'autres pratiques courantes s'adressent plutôt aux autorités locales: les grèves des cours et les " grèves de la faim ". Après les expulsions de 1961, par exemple, les étudiants africains font grève des cours et grèves de restaurant universitaire. La grève de "restau u », dans la mesure où ils et elles ne mangent jamais qu'au « restau u » équivaut de facto à une grève de la faim et est pensée comme telle. Ces grèves ne durent pas et ont un caractère plus symbolique que vraiment opératoire ${ }^{11}$.

Il est toute une gamme d'autres pratiques que nous qualifierons de "positives ", en opposition aux pratiques "négatives " de réaction et défense, sans qu'il y ait là de jugement de valeur. On peut distinguer quatre ensembles: la diplomatie, les pratiques culturelles, les pratiques ou stratégies d'alliance, la propagande. Ce sont quatre ensembles qui, parfois, se chevauchent.

\section{Diplomaties}

On sait ce que les décolonisations doivent aux changements internationaux, aux rapports de force de la guerre froide ou à la tribune des Nations Unies. Les étudiants africains en France l'ont parfaitement compris. Ils multiplient les voyages (Dieng 2011 ; Camara 2010), affirmant la FEANF dans de multiples instances, et dans des conditions d'autant plus propices que l'Est comme l'Ouest désirent se concilier les futures élites de l'Afrique. II n'y a pas là seulement désir de voyages, mais un souci de reconnaissance. La scène internationale confère une légitimité à la revendication d'indépendance et l'aspiration au socialisme dont sont porteuses les délégations de la FEANF. Ces très nombreux voyages se poursuivent après l'indépendance dans un contexte d'offres démultipliées par la concurrence induite par la Guerre froide, et par la querelle sino-soviétique, et donc le souci des camps antagonistes de se rallier ce qu'on appelle alors, depuis la Conférence de Bandung en 1955, le "Tiers monde ". Ils témoignent aussi d'une mondialisation des oppositions, d'une circulation planétaire de pratiques militantes, qu'elles soient syndicales ou étudiantes/intellectuelles. Le voyage en Chine [fig.2], essentiellement après 1962, remplace ou co-existe avec le voyage en URSS et les dirigeants de la FEANF assistent aux défilés de garde rouges et serrent la main de Mao-Tse-Toung 
comme ils serrent celle d'Enver Hodja, d'autant plus que l'Albanie fait office, après l'adoption par la Chine de la théorie des Trois-mondes ${ }^{12}$, de dernier sanctuaire du socialisme. Sont également invités aux congrès de la FEANF des délégués des étudiants chinois, vietnamiens, albanais, ou des représentants des Départements d'Outre-Mer (DOM).

\section{Stratégies d'alliance}

Les alliances ne se cultivent pas seulement au niveau international. II en est une, en France même, qui s'est révélée éminemment stratégique. C'est l'alliance, plus ou moins opportuniste selon les cas, avec le Parti communiste, d'une certaine façon un allié naturel. Quelques étudiants de la FEANF sont adhérents au parti, tels Benoît Ondua Balla, Amady Aly Dieng, Ousmane Camara, Babacar Niang, Seyni Niang, sans être toujours d'accord avec sa politique coloniale. Ils se réunissent au sein d'un " groupe de langues ${ }^{13}$ dépendent de la section des provinces de France, sise à la Cité Universitaire du boulevard Jourdan. La FEANF bénéficie de l'aide matérielle du PCF et de la CGT : prêt de salles par exemple, comme celles de la Maison des métallos, rue Jean-Pierre Timbaud, où celles de la rue Gît-le-Cœur, où s'ouvrent la plupart des congrès, ou aide financière directe ${ }^{14}$. L'aide peut passer par le Secours populaire. Elle passe par les services rendus par deux avocats communistes, Maîtres Vergès et Kaldor. Mais le primat des étudiants africains est la question nationale, et non la question des classes. Les étudiants connaissent la Lettre à Maurice Thorez d'Aimé Césaire, par laquelle ce dernier rompt de manière retentissante avec le PCF après le rapport Khrouchtchev. Néanmoins, quelles que soient les divergences plus ou moins grandes avec le PC, le primat du marxisme s'impose peu à peu dans la direction de la FEANF. En 1957, l'étudiant Majhemout Diop crée à Thiès le Parti africain de l'indépendance (PAI) qui se réclame du marxisme, appelant de ses vœux dans son manifeste fondateur une Afrique indépendante et socialiste. La majorité des membres du CE de la FEANF adhèrent au PAI. C'est en fait le PAI qui y « fait » les élections. Après la rupture sino-soviétique, les rapports de la FEANF avec le PC et la CGT se tendent, l'état-major de I'organisation se retrouvant majoritairement maoïste à partir de $1965^{15}$. Le PAl lui-même éclate et émergent des Groupes marxistes-léninistes (GML) qui s'opposent à un parti resté «communiste orthodoxe ». Si cette fracture touche la fédération, elle n'épargne pas non plus les associations nationales qui en sont adhérentes, dont certaines, telle l'Union Nationale des Etudiants du Kameroun (UNEK), se scindent. Le PC et la CGT continuent à dispenser une aide parcimonieuse mais réelle, tandis que l'état-major de la FEANF se perd dans des débats théoriques sans fin. Ce qui manque beaucoup plus cruellement, c'est le soutien financier des États africains. Le coup d'État d'Houari Boumediène, en 1965, met fin à celui de l'Algérie et celui de 1966 à celui du Ghana, qui avait en outre offert à la fédération un hôtel particulier à Neuilly. Seul le Congo socialiste envoie encore de 
maigres subsides. Les autres États, qui sont autant de cibles pour les étudiants, cessent les versements des territoires avant les indépendances.

\section{Pratiques et/ou stratégies culturelles}

La FEANF n'a cessé de promouvoir les cultures africaines sur le territoire français. Certains de ses dirigeants avaient d'ailleurs déjà entamé des actions de promotion culturelle via des associations créées en Afrique, avant leur départ pour la France. Ousmane Camara avait, par exemple, fondé au tout début des années 50, une association de jeunesse du Siné-Saloum, dotée d'un journal : Lien culturel. En France ces pratiques, qui sont aussi une façon de répondre à l'imposition de la culture française, sans d'ailleurs récuser cette dernière à condition qu'elle n'écrase pas la culture originelle mais coexiste avec elle, prennent diverses formes: expositions, pièces de théâtre, ballets. Les expositions sont montées avec des objets venus dans les bagages des étudiants ou avec, véritable ironie de l'histoire, des pièces empruntées au Musée de l'homme. Les ballets acquièrent parfois une renommée conséquente. C'est le cas notamment de la troupe du guinéen Fodeba Keita, qui deviendra plus tard ministre dans la Guinée de Sékou Touré pour finalement succomber à la sanglante répression orchestrée par ce dernier. Danses et pièces de théâtre animent les « Nuits de I'Afrique " [fig.3 et 3bis] régulièrement organisées lors des festivités de la semaine anti-coloniale, autour du 21 février $^{16}$. Par exemple, en 1964, 800 personnes se sont réunies à la maison des métallos rue Jean-Pierre Timbaud et après une brève allocution d'Ibrahima Ly assistent à plusieurs ballets africains, puis un bal a clos la soirée ${ }^{17}$. Les étudiants jouent des pièces empruntées au répertoire existant, tel Le Roi Christophe d'Aimé Césaire qui connait un succès triomphal ${ }^{18}$, ou en écrivent pour les besoins de la cause. Les étudiants centrafricains composent collectivement une pièce au vitriol contre Bokassa, Le commencement de la fin $^{19}$. Cette pratique n'est évidemment pas née avec le séjour en France car le théâtre est présent de longue date sur les terres africaines, dans un but tant festif que promotionnel.

A côté de la promotion des cultures africaines, on pourrait inclure dans les pratiques ou stratégies culturelles l'apprentissage du marxisme, objet d'un investissement très sérieux : pour les membres de la FEANF qui sont aussi au PAI, les cours du Centre d'études et de recherches marxistes (CERM) sont obligatoires. Au niveau supérieur, on est prié de suivre ceux de l'université nouvelle. Cela n'empêche pas non plus le travail sur les grands textes menés dans le cadre de cercles d'études marxistes, animés par les étudiants. Ainsi à Toulouse, un cercle d'études marxistes, animé par Joseph Van Den Reysen [fig.6] se réunit régulièrement dans un grand café de la place du Capitole ${ }^{20}$. Amady Aly Dieng centre son propre groupe d'études sur Le Capital. Ce groupe sera d'ailleurs prorogé après le retour d'Amady au Sénégal (Dieng 2011). Le marxisme est pris très au sérieux et va parfois servir 
de principe de gouvernement après le retour. Simon Ifede Ogouma par exemple ou François Codjo Azodogbehou [fig4] sont parmi les conseillers de Kérékou qui inspirent au chef d'état dahoméenbéninois le choix du marxisme-léninisme comme science de gouvernement ${ }^{21}$. Bien des socialismes africains sont ainsi inspirés par d'anciens étudiants que leur séjour en France a imprégnés de ce qu'ils considèrent comme une forme de contre-culture, alternative à la culture dominante, et coloniale.

\section{Propagandes}

Il est de multiples moyens de propagande utilisés par la FEANF. En France même, outre les diverses conférences et journées d'études où interviennent des leaders de la FEANF ou des intellectuels amis de l'Afrique tels Suret-Canale, Edouard Glissant ou Roger Garaudy ${ }^{22}$, la Fédération met en place des cours d'alphabétisation des travailleurs africains, à Saint-Denis par exemple, sous l'impulsion d'Emmanuel Ngassa à partir d'octobre 1964 ${ }^{23}$. Des militants, tel Robert Dossou, organisent les travailleurs. Dossou, le président fondateur de l'association des travailleurs dahoméens ${ }^{24}$, est régulièrement allé en province, en compagnie de Valentine Quenum, visiter les diverses communautés.

En Afrique, la propagande active que menait la FEANF, sous forme de cours, conférences etc, est devenue plus difficile après les indépendances. Les nouveaux Etats se montrent plus sévères que les autorités coloniales. L'université populaire fondée par I'UGEAO (Union générale des étudiants d'Afrique de l'Ouest) en septembre 1956 perdure au Sénégal mais il est devenu impossible de faire des conférences plus politiques, sinon dans des cas très exceptionnels, comme au Congo socialiste. De même, la diffusion de l'Etudiant d'Afrique noire est contrariée. Nous n'avons plus de chiffres après 1960. Entre difficultés financières récurrentes et censure, il est bien possible que la diffusion ait cessé ou ait été , en tous cas, fortement limitée.

\section{TRAJECTOIRES}

Les étudiants d'Afrique sub-saharienne en France ont, de l'immédiat après-deuxième guerre mondiale au début des années 80 , des propriétés partagées qui permettent de les penser comme un groupe social : spécificités en termes de disciplines avec dominantes fortes de la médecine et du droit au début de la période ; gestion par un même organisme - l'Office des étudiants d'Outre-mer (OEOM) devenu Office central de coopération universitaire (OCAU) en $1962^{25}$ - ; spécificités en termes d'âge - plus élevé que la moyenne des étudiants en France; spécificités en terme de situation matrimoniale - ils sont plus souvent mariés; 
spécificités en termes de genre - les femmes sont en grande minorité ; et spécificités en termes de statut puisqu'ils sont, au moment où est créée la FEANF, citoyens de I'Union française, puis, après 1960, étrangers, mais pouvant acquérir la nationalité française par simple déclaration et bénéficiant encore d'avantages théoriquement réservés aux citoyens français - liberté de circulation et d'établissement jusqu'au mitan des années 70 (Blum 2016). Ils sont aussi objet d'enquêtes (Sondages 1961; N'Diaye 1962) ${ }^{26}$. Ceci dit, des tendances centripètes menacent, tout au long des années 70 , la cohérence du groupe : profil disciplinaire tendant à rejoindre celui de l'ensemble des étudiants en France [figA], montée des nationalismes, évolution des statuts dans le sens d'une assimilation aux autres étrangers etc.... Le comité exécutif présente le reflet à peu près fidèle de ces spécificités et évolutions. Les origines sociales sont aussi extrêmement diverses comme pour l'ensemble des étudiants africains : l'école française a bouleversé les hiérarchies originelles et a, d'une certaine façon, , mis les compteurs à zéro. Pour n'en donner qu'un exemple on rappellera celui bien connu de l'École des otages, devenu École des fils de chef et qui donnera naissance à William Ponty. Les colonisateurs avaient fait obligation aux chefs d'envoyer leurs enfants. Mais ceux-ci, peu soucieux de se plier aux désirs de l'occupant, avaient contourné l'ordre en envoyant les enfants d'esclaves qui, traditionnellement, portaient le même nom. Les modes de sélection des enfants envoyés à l'école ont été très variés selon les territoires, selon aussi la présence ou non de missions et d'enseignements religieux, et les contournements tout aussi variés. II faudra attendre le milieu des années 60 ou le début des années 70 pour que de nouveaux phénomènes de reproduction se mettent en place ${ }^{27}$. La figure $B$ donne quelques indications sur cette diversité sociale mais ne permet pas de prendre en compte le rôle de positions sociales plus " traditionnelles ${ }^{28}$. L'appartenance aux castes, par exemple, est extrêmement difficile à saisir, car un individu ne se dira jamais casté. On parlera des castes ou des castés mais jamais on ne formalisera les données à ce sujet par écrit. Ces statistiques ne disent rien non plus sur les ascendances nobles ou royales, ou au contraire serviles, bref, elles ne disent rien des typologies sociales en vigueur en Afrique, où existent parfois de très fortes endogamies. Elles ne disent pas si le père, simple fonctionnaire des postes, par exemple, n'est pas aussi un grand lettré musulman de famille royale. Malgré tout, ces enquêtes montrent une réelle diversité de recrutement des étudiants, ce que permet l'octroi systématique des bourses aux bons élèves. Les années 60 vont être aussi celles où les hiérarchies du diplôme vont cohabiter avec les hiérarchies sociales originelles. Un casté va pouvoir devenir ministre mais cela peut encore poser problème et le discréditer. 
Toujours est-il qu'incontestablement l'exil gomme les différences sociales, d'autant que les critères ne sont pas les mêmes d'un bout à l'autre de l'Afrique, et consolide les solidarités devant une même situation.

Il y a aussi, au CE, une surreprésentation des Sénégalais avant 1960 et des Dahoméens après 1960, une présence constante des Congolais (Congo-Brazzaville) et au tout début une présence très forte des Togolais et Camerounais destinée à éluder le conflit toujours menaçant entre Sénégalais et Ivoiriens. Il faut noter aussi que la part des femmes au CE de la FEANF est très faible : 4,3\% sur l'ensemble de la période considérée.

Pour beaucoup la FEANF n'avait été que la suite logique d'investissements militants déjà engagés avant l'arrivée en France. Dans les grands lycées de l'AOF ou AEF, Robert Dossou, Bruno Amoussou, Ousmane Camara, Amady Aly Dieng, Jean-Baptiste Oualian entre autres avaient participé ou initié de grandes grèves, la plupart du temps destinées à protester contre une alimentation médiocre et, surtout, venue d'ailleurs. Ceux et celles qui étaient d'abord passés par l'université alors régionale de Dakar avaient milité à I'AGED (Association générale des étudiants de Dakar) ou à I'UGEAO, comme Tidiane Baïdy Ly, Ousmane Camara, Charles Diané, Moustapha Diallo. Ils ou elles avaient donc une carrière militante parfois longue. Ce double capital, savant et militant, a au moins partiellement déterminé une agency ultérieure. Nombre d'entre eux vont être d'actifs protagonistes des constructions nationales, par leur pratique professionnelle ou politique. Ils auront pour certains des postes de responsabilité - premier ministre comme Habib Thiam, directeur général de l'UNESCO comme Ahmadou Mahtar Mbow, directeur général adjoint du BIT comme Albert Tévoédjré ou Président de la République comme Alpha Condé. "L'école nouvelle» bouleverse les hiérarchies traditionnelles, propulsant un fils de paysans comme Olabihy Yaï au conseil exécutif de I'UNESCO mais ravalant aussi, a contrario, un fils de roi comme Jean-Baptiste Oualian à un poste d'exécutant. Dans ce que l'on peut bien appeler la génération FEANF, dans la mesure où de 1950 à 1976 I'horizon d'attente est le même, l'indépendance ou une autre indépendance, peu restèrent en France, si ce n'est cas de force majeure.

On ne donnera ci-après que quelques exemples de trajectoires parfois étonnantes, du village de brousse aux plus hautes responsabilités politiques. Il s'agit de savoir à quelles positions de pouvoir une élite étudiante, de surcroît militante, est arrivée, postérieurement au premier engagement. Et à l'inverse, de scruter les situations les plus tragiques auxquelles le destin ou plutôt les soubresauts de la vie politique en ont conduit d'autres. Nombreux sont ces étudiants à avoir été à un moment ou l'autre de leur carrière ministres dans leur pays. L'un, Alpha Condé, a même été et est encore, au moment où on écrit ces lignes, Président de la République de Guinée. Le tableau suivant décrit l'accession aux plus hautes responsabilités politiques et à l'inverse les situations de rupture : 


\begin{tabular}{|l|l|c|l|}
\hline CE & Effectif du pays & $\begin{array}{c}\text { Exil, prison ou } \\
\text { mort violente }\end{array}$ & Ministres \\
\hline Total & $\mathbf{1 9 5}$ & $\mathbf{2 7}(\mathbf{1 3 , 8 4 \% )}$ & $29(14,87 \%)$ \\
\hline Cameroun & 11 & $\mathbf{3}$ & 1 \\
\hline Congo-Brazzaville & 24 & $\mathbf{3}$ & 6 \\
\hline Ivoiriens & 6 & $\mathbf{0}$ & 0 \\
\hline Dahomey-Bénin & 31 & $\mathbf{4}$ & 8 \\
\hline Gabon & 5 & $\mathbf{0}$ & 0 \\
\hline Guinée & 19 & $\mathbf{8}$ & 1 \\
\hline Hte-Volta/Burkina & 18 & $\mathbf{1}$ & 1 \\
\hline Mali & 7 & $\mathbf{2}$ & 0 \\
\hline Mauritanie & 3 & $\mathbf{0}$ & 0 \\
\hline Niger & 8 & $\mathbf{0}$ & 0 \\
\hline RCA & 7 & $\mathbf{0}$ & 1 \\
\hline Sénégal & 41 & $\mathbf{4}$ & 8 \\
\hline Tchad & 2 & $\mathbf{0}$ & 1 \\
\hline Togo & 13 & $\mathbf{2}$ & 2 \\
\hline
\end{tabular}

On constate que le pourcentage de ministres le plus élevé est celui du Congo-Brazzaville, suivi par le Dahomey et le Sénégal. Au Congo, cela s'accompagne d'une longévité du personnel politique, mais aussi de nombreuses allées et venues. Les crises politiques que connaît le pays - révolution des Trois Glorieuses, prise de pouvoir par Marien Ngouabi, tentatives de putschs, assassinats politiques, guerre civile - entraînent bien entendu des ruptures dans la vie des individus de même que dans leurs carrières politiques (Bazenguissa 1997). Cela n'empêche pas des formes de longévité, mais avec des éclipses, des retraits et des retours. Au Dahomey, ce ne sont pas les mêmes qui sont ministres sous le régime marxiste de Kérékou et après la Conférence nationale des forces vives de la nation, en 1990. Il y a nettement rupture. Le Sénégal, quant à lui ne connaît pas de crises majeures, ni révolution, ni guerre comme au Congo, ni prise de pouvoir par les militaires - et seulement deux présidents entre 1960 et 2000 . Les carrières politiques peuvent être, dans ces conditions, fort longues, et conditionnées plus par des formes d'affinités personnelles que par des transformations politiques.

On peut regarder maintenant, à l'inverse, les situations de rupture : exil, prison et, à l'extrême, mort violente. La palme revient incontestablement à la Guinée, et le régime de Sékou Touré est, sans nul doute, le plus répressif pour les anciens dirigeants de la FEANF. Dans le groupe des dix-neuf Guinéens ayant appartenu au $\mathrm{CE}$, cinq sont " morts à Camp Boiro ", ce qui représente un taux de mortalité violente considérable par rapport à l'ensemble du CE. Le « Camp Boiro » désigne un camp militaire au sein duquel se trouvait une prison politique, situé à Conakry. Mais l'expression renvoie, plus largement, à l'ensemble des lieux de détention, d'exécution et d'inhumation des prisonniers politiques. Sont morts à « Camp Boiro » Abdourahmane Bah (CE de 1960 et 1961), Aliou Thierno Bah 
(CE de 1962 et 1964), Mamadou Barry (CE de 1959), Kemoko Keita (CE de 1961), N’Ki Traore (CE de 1951-1952), Souleymane Sy Savané ( CE de 1955). D’autres ont échappé à la mort par l'exil comme Sékou Traoré au Congo-Brazzaville, Charles Diané au Gabon, tous deux condamnés à mort par contumace, ou Alpha Condé qui, lui aussi condamné à mort par contumace, en 1970, reste en France où il travaille comme maître assistant en droit et sciences politiques à l'Université PanthéonSorbonne de 1968 à 1975. Cela en dit long sur le sort réservé par la Guinée de Sékou Touré à ceux qui auraient pu en être, ou en furent éphémèrement les cadres.

C'est donc un groupe dont un pourcentage non négligeable des membres a accédé à de hautes responsabilités, mais dont un autre pourcentage non moins négligeable a pu connaître exil, prisons ou assassinats. Les deux ne sont pas exclusifs : on a pu connaître exil et/ou prison et devenir ministre dans des circonstances politiquement plus favorables. C'est le cas par exemple d'Alpha Condé, encore lui, qui connut exil, prison puis accéda aux sommets de l'État; Bruno Amoussou qui connut la prison puis l'exil durant le régime militaro-marxiste de Mathieu Kérékou (Amoussou 2009) ${ }^{29}$; Noé Kutuklui qui fut ministre dans un éphémère gouvernement Grunitiski, au Togo etc...

A ces données générales correspondent des trajectoires de vie, que l'on a pu documenter à I'aide d'entretiens menés en France mais aussi au Sénégal, au Congo-Brazzaville et au Bénin. Le type de reconversion accomplie dépend aussi des régimes en place, et des temps de la vie. Un même individu peut, selon les différentes étapes de sa vie, et les évolutions politiques à l'intérieur d'un continent africain soumis alors à de multiples bouleversements, qu'il s'agisse de coups d'Etat ou de révolutions, ressortir successivement à plusieurs de ces catégories. Les itinéraires sont mouvants au sein d'une cité politique qui l'est aussi. Très peu sont restés en France, dans ces années charnières, qui sont aussi celles où l'on croit encore à une grande cause : la fabrique de la nation africaine, et où I'on vit avec le sentiment que tout est encore possible, apanage heureux des années 68. Cela changera, avec les désillusions des années 70.

On en propose ici une catégorisation réalisée de façon inductive, à partir de quelques exemples de figures marquantes.

\section{Ceux qui sont restés ou se sont retrouvés dans une opposition radicale}

Le Camerounais Osende Afana (Diané 1971) est assassiné en 1966 dans les maquis du Cameroun (Deltombe 2011 ; Mbembé 1991). II avait été rédacteur en chef de l'Etudiant d'Afrique noire, et trésorier de la FEANF. Il est membre de l'Union Nationale des Étudiants Kamerunais (UNEK) et de I'Union nationale des Populations du Cameroun (UPC), qui, depuis 1955, mène la guerre du Cameroun contre les Français, puis à partir de 1960 contre le gouvernement d'Ahmadou Ahidjo. 
Osende Afana quitte la France en 1958 pour rejoindre la direction de I'UPC en exil, au Caire. II devient, après l'assassinat dans les maquis camerounais de Ruben Um Nyobe, le représentant de I'UPC au secrétariat afro-asiatique du Caire. II est membre de la délégation qui défend I'indépendance du Cameroun devant l'assemblée des Nations Unies. II quitte le Caire pour Conakry quand I'UPC installe son siège en Guinée. II appartient au Comité révolutionnaire constitué après le meurtre de Félix Moumié (Garbely 2005). Il y a aussi dans ce comité un autre membre du CE de la FEANF, Michel NDoh. Puis Osende Afana décide de rallier le maquis, peut-être suite à des dissensions avec les Soviétiques, en particulier avec l'ambassadeur soviétique au Caire, ce dont témoignent quelques lettres retrouvées à Moscou ${ }^{30}$.

Michel Ndoh, quant à lui, expulsé de France en 1961, se réfugie au Ghana. Quand un coup d'état militaire renverse Nkrumah en février 1966, lui et Jean-Martin Tchaptchet [fig.5], sont emprisonnés par le nouveau pouvoir par « mesure de protection ». Maître Kaldor réussit à les faire sortir. On craint un moment que les deux hommes aient été extradés vers le Cameroun, ce qui signifiait une mort certaine pour ces deux leaders de l'UPC. Finalement, il n'en est rien. Ils sont toujours au Ghana mais interdits de travailler et dans une situation de mobilité restreinte. Michel Ndoh se retrouve néanmoins assez vite exilé en Italie, qui a accepté de le recevoir et ce toujours grâce à l'aide de Kaldor et du Haut-Commissariat aux réfugiés des Nations-Unies qui prend en charge les frais du voyage. C'est d'Italie qu'il adresse au Président Georges Pompidou une lettre où il demande l'asile politique à la France, arguant du fait qu'il s'est fait l'agent de diffusion de la langue et culture française au Ghana, en donnant des cours ${ }^{31}$. On ne sait malheureusement pas ce qu'il advint de lui ensuite.

\section{Ceux qui ont gardé une posture critique}

Garder une posture critique - et publique - n'est pas toujours possible. Cela le fut dans le Sénégal de Senghor, même s'il ne fut pas un modèle de démocratie. Mais l'arbitraire absolu, pour ne pas dire la farce sanglante qui se joua dans certains pays africains épargna malgré tout le territoire : sagesse du poète-président peut-être, mais aussi histoire singulière au sein de l'Empire avec les Quatre Communes ${ }^{32}$, et leurs citoyens français: le racisme colonial y sévit peut-être moins violemment qu'ailleurs, qu'en Afrique centrale par exemple, où régnèrent Bokassa ou au Togo Eyadéma, qui tous deux servirent de modèle au romancier Ahmadou Kourouma (1999).

Boubakar $\mathrm{Ly}^{33}$ milita activement pour sa discipline, la sociologie. D'une famille originaire du Fouta, il a commencé ses études secondaires au lycée Van Vollenhoven de Dakar mais les continue à Saint-Maur, au lycée Marcelin Berthelot où a enseigné Senghor. II fait partie de la première génération d'étudiants à avoir suivi un cursus autonome de sociologie, il rentre à Dakar en octobre 
1967. Il est le premier Africain à y obtenir un poste d'assistant en sociologie. Mais viennent les évènements de 1968. II est arrêté, lors de la grande rafle à la Bourse du travail. II passe une dizaine de jours en prison, à Thiès puis Dakar. Ses collègues réussissent à le faire sortir assez vite en prétendant qu'il effectuait une enquête sociologique. Après 1968, le département de sociologie est supprimé par l'État, qui le juge trop subversif. Boubakar Ly fait alors du maintien de la sociologie son combat. Outre à l'université, il continue à l'enseigner dans diverses écoles. Son acharnement n'est pas vain puisqu'en 1992, une section de sociologie est rouverte au département de philosophie et en 1994, un département est recréé. Il le dirige pendant deux ans, de 2000 à 2002.

\section{Ceux qui sont restés dans l'opposition partisane ou syndicale}

La situation est très complexe pour le dahoméen-béninois Gédéon Dassoundo ${ }^{34}$, fils de paysans méthodistes, et ce, du fait de la confusion qui a régné dans le Bénin marxiste-léniniste. C'est un opposant, ce qui ne l'empêchera pas d'être quelque temps ministre de Mathieu Kérékou. Le gouvernement marxiste-léniniste issu du coup d'État militaire du 26 octobre 1972 a besoin des cadres, et des diplômés, et mène une politique de carotte et de bâton pour se les concilier, voire les contraindre à participer. C'est ce qui arrive à Gédéon Dassoundo. Rentré de France avec un diplôme de statisticien en poche, il est nommé au service de la statistique. Il devient vite secrétaire général, puis président de l'association des jeunes de la sous-préfecture de Dassa, dont le principal objectif est le travail auprès des paysans. En 1973, il participe à la création du premier syndicat paysan, sur le modèle chinois, et, en 1974, à celle de la fédération des organisations de jeunesse qui ne vivra que de janvier à avril, mois au cours duquel toutes les associations de jeunesse sont dissoutes et interdites par le pouvoir, à l'exception de la Ligue nationale de la jeunesse patriotique. A l'égard du pouvoir, l'OMLD (Organisation des Marxistes-léninistes du Dahomey) est divisée. Certains plaident la confiance, d'autres dont Gédéon Dassoundo, ne croient pas pouvoir donner la leur à des militaires. Cela ne l'empêche pas, avec ses amis politiques, de participer au brain-trust convoqué pour réfléchir à ce qui va devenir le discours-programme ${ }^{35}$, mais dans une commission alternative à celle qui a été officiellement mise en place : la commission nationale spéciale.

Cette posture critique, à la fois dedans et dehors, de Gédéon Dassoundo s'éclaire à l'aune de la pratique du pouvoir sous Kérékou, entre répression et concertation, stratégies diverses d'alliance avec ses opposants, conciliations et retours de bâtons, intégration plus ou moins forcée des cadres, suivant les nécessités de l'heure. Toujours est-il que les diverses commissions et conférences mises en place durant la période marxiste-léniniste du Bénin ont pu être autant de préludes inaboutis à la première conférence nationale en Afrique : la Conférence des forces vives de la nation en 1990, qui, cette fois, tient ses promesses. 
Gédéon Dassoundo participe à la conférence des cadres, convoqué en 1979 par le régime, et où la parole s'exprime très librement. Mais, ce qui est plus surprenant, il est appelé avec un de ses compagnons d'opposition à rejoindre le gouvernement comme ministre, ce qu'il doit accepter, bon gré mal gré.

GD : Donc nous avons refusé [d'entrer au gouvernement]. En 82, le 9 avril 1982 le gouvernement a été annoncé. Un nouveau gouvernement, qui a été formé, a été annoncé, dans lequel j'étais ministre de l'Agriculture, ministre du Développement rural et de l'action coopérative on disait à l'époque. Un autre a été nommé ministre des Sociétés d'État. Nous avons refusé, ils nous ont contraints de rester en poste, nous avons négocié pendant huit mois,...1

Quand enfin, il est "libéré ", il fait l'objet de multiples persécutions dans son travail, qui l'obligent à prendre une retraite anticipée et à créer un cabinet de consultance, qu'il sera aussi forcé d'abandonner pour cause de harcèlement fiscal. Mais la Conférence nationale des forces vives de la nation porte bel et bien ses fruits et annonce une "transition démocratique " à laquelle Mathieu Kérékou a la sagesse de ne pas s'opposer.

\section{L'accommodation}

On désigne par là, à la suite de Philippe Burrin (1995), l'attitude de ceux qui ont " fait avec » des régimes qu'ils avaient parfois combattus. Ils ne se sont pas compromis, sans que l'on sache toujours quel type de rapports ils entretenaient avec le politique: adhésion plus ou moins convaincue, rejet non exprimé dans la sphère publique, attente de lendemains meilleurs ou plus adaptés à leur désir d'investissement, voire indifférence. Cela concerne notamment ceux qui s'investirent dans leur profession, sans plus d'activités politiques, du moins avant le retour à la démocratie. On peut ranger dans cette catégorie aussi bien des universitaires (le burkinabe Bakary Coulibaly, le sénégalais Abou El Caba Touré), des juristes ou avocats (le sénégalais Mamadou Lô), des médecins (le sénégalais Moustapha Diallo), et des ingénieurs, des banquiers (l'ivoirien Abdoulaye Fadiga par exemple gouverneur de la BCEAO), des fonctionnaires internationaux (le dahoméen Albert Tévoédjré).

Si I'on reste au Dahomey-Bénin de Mathieu Kérékou, Valentine Quenum [fig.7] s'est accommodée du régime jusqu'à un point de rupture. Elle était étudiante à Dakar lors du « Mai sénégalais » et alla ensuite poursuivre ses études en France. En effet, lors de la révolte étudiante de Mai 68 à Dakar, Senghor avait expulsé tous les étudiants étrangers, rapatriés par avion dans leur pays d'origine. La France offrit alors des bourses dont profitèrent certain-e-s des expulsé-e-s dont Valentine Quenum ou le voltaïque Jean-Baptiste Oualian, pour ne citer que des membres du CE de la FEANF. Valentine Quenum est devenue, après son retour au Dahomey en 1973, secrétaire générale d'un Comité neutre des femmes qu'elle a contribué à mettre en place par opposition à d'autres 
organisations de femmes, plus politisées. Elle représente le Comité neutre au Conseil National de la Révolution (CNR). Mais elle n'y est pas à l'aise, n'en partageant pas, souvent, les points de vue. La rupture décisive pour elle vient quand le CNR, transformé en tribunal, juge un certain nombre d'accusés dont son oncle, le père Alphonse Quenum. Tous sont condamnés à mort. L'expression de son désaccord avec la sentence suscite la méfiance des autorités révolutionnaires et une surveillance, alors même qu'une partie de ses proches lui en veulent pour cette condamnation. La condamnation à mort n'est pas exécutée : les accusés font dix ans de prison. Mais Valentine Quenum est échaudée par cette expérience : quand l'Assemblée nationale révolutionnaire (ANR) se met en place en 1984, elle part dans le Nord du pays pour être sûre de ne pas y être déléguée ${ }^{37}$.

Certains ont, une fois rentrés au pays, rallié des régimes qu'ils conspuaient lors de la vie étudiante et militante. S'il ne s'agit évidemment pas ici de juger, il serait intéressant de comprendre selon quelles modalités se fait ce type d'évolution. On n'en a malheureusement pas toujours les moyens, faute de matériaux suffisants ou faute d'avoir pu réaliser les entretiens nécessaires.

\section{Ceux qui se sont compromis}

BL: Beaucoup de camarades, dès qu'ils sont rentrés, se sont empressés d'aller au pouvoir. Le cas d'Ousmane Camara, entre nous, a été critique, car tout de suite il a été nommé à un poste de responsabilité, au niveau de sécurité, qui lui a permis de connaître les secrets du parti, il savait où se trouvaient les uns et les autres, on a été simplement les cueillir.... Les gens ont estimé à un moment donné qu'ils devaient aller au pouvoir, des gens qui avaient été de très bons militants anti-colonialistes. Je vous ai parlé de beaucoup de trahisons de tout bord - trahisons, non - disons déviations par rapport à la ligne. Pour d'autres pays je peux citer des cas, c'est Lopès ${ }^{38}$ qui a été candidat. C'est surtout celui qui a été le Premier ministre de Bokassa, Patassé, c'est le cas typique. Il a été le Premier ministre de l'empereur. Un ancien militant de la FEANF et du PAl, qui s'est retrouvé premier ministre pendant la période de l'empire, vous voyez à quel point : Le goût du pouvoir. Il faut dire, il faut peut-être expliquer cela, même si ça ne justifie pas. Quand les uns et les autres sont rentrés au pays, c'était l'absence totale de démocratie, il n'y avait plus de référence, il n'y avait plus d'organisation, les gens se sont trouvés livrés à eux- mêmes, et au Sénégal en tout cas il y a eu des pressions familiales, les gens se sont retrouvés avec leur famille, leur région, leur ethnie, il y a eu des pressions.....C'est pourquoi nous autres nous ne condamnons pas beaucoup de nos camarades qui à l'époque étaient au pouvoir ${ }^{39}$.

Ousmane Camara, né à Djourbel d'une famille bigame de 6 enfants dont le père est mécanicien, avait commencé ses études supérieures à Dakar, où il milita déjà activement au sein de I'Union générale des étudiants d'Afrique occidentale (UGEAO), avant de venir à Paris. Une fois rentré au Sénégal, en effet, il devient magistrat et procureur (Camara 2010). C'est lui qui instruit le procès de Mamadou $\operatorname{Dia}^{40}$, pour lequel il requiert d'ailleurs un verdict plus clément que celui qui finalement envoya le président du conseil en prison. Le problème est qu'Ousmane Camara, très au fait par son passé politique des secrets d'un PAI désormais clandestin, est amené à instruire des affaires où des membres du PAI sont impliqués. Ousmane Camara, outre ses fonctions à la justice qui le mènent jusqu'à la présidence de la cour suprême, est au moment des évènements de mai 1968 directeur de 
la sûreté. Il a eu aussi sous Senghor une brillante carrière ministérielle. Mais Ousmane Camara ne voit aucune contradiction entre ses activités de jeunesse et celles de l'âge adulte. Pour lui, une fois l'indépendance acquise, il fallait construire la nation. Et il a voulu participer à cette construction.

II faut pour mieux comprendre, au-delà des explications unificatrices, considérer les trajectoires personnelles, où l'affect joue incontestablement un rôle de même que les trajectoires politiques des États, et la négociation qui se mène entre les deux. Quelquefois l'amitié a survécu aux oppositions politiques comme c'est le cas de celle qu'entretiennent Amady Aly Dieng et Ousmane Camara : ils ont continué à cultiver la leur malgré des choix politiques opposés. C'est Habib Thiam, sans aucun doute qui fournit le meilleur exemple de ce rôle de l'affect. Il a été deux fois Premier ministre sous la présidence d'Abdou Diouf, acceptant sans acrimonie d'être renvoyé une première fois pour les besoins de la politique et de revenir quand Abdou Diouf le rappelle. S'il accepte de servir l'Etat sénégalais c'est, écrit-il, par amitié envers quelqu'un qu'il a côtoyé à la Cité universitaire du boulevard Jourdan et sur les bancs de l'ENFOM :

" J'appartiens à une génération de cadres sénégalais sortis des universités françaises lorsque mon pays accédait à l'indépendance. En ces temps, les perspectives et possibilités de carrière étaient nombreuses et fort attrayantes... Seulement le contexte politique et notre engagement aux côtés de nos aînés dans le combat pour l'émancipation des peuples colonisés avaient fait de nous des militants acharnés mais lucides d'une dynamique au bout de laquelle nos pays devraient aller vers l'indépendance. Je suis rentré au Sénégal, en totale harmonie avec les idées qui fondaient ma réflexion et pour lesquelles, aux côtés d'autres camarades, je m'étais battu dans les organisations syndicales estudiantines. Avant, et bien avant la politique, il y avait les amitiés. Celles d'abord nouées au pays et celles qui naquirent sur les campus universitaires, sur les bancs des facultés et des écoles de formation.... Au nombre de celles-là, il y a l'amitié qui me lie encore à Abdou Diouf.» (Thiam $2001: 9$ )

II faut aussi scruter attentivement, au cas par cas, ce qui dans l'art de gouverner emprunte à l'art de s'opposer, dans lequel étaient passés maîtres les hommes et les femmes de la FEANF. Quelle que soit la tribune, l'art de la tenir est le même. Un capital culturel et scolaire, hybride dans les cas ici analysés, puisqu'il emprunte à des cultures différentes, prédispose à de hautes responsabilités. La politique conduit à des manières d'être et de faire qui se reconvertissent aisément de l'opposition au pouvoir, et ce d'autant plus que l'on peut passer d'une cause à l'autre sans avoir forcément l'impression de se renier : de l'utopie d'une Afrique socialiste et unie à une très pragmatique construction nationale. D'un nationalisme rêvé à un nationalisme de fait.

On peut parler d'une "génération FEANF ", au sens où Mannheim entend génération (Mannheim, 2005). Une analyse plus fine permettrait d'ailleurs de distinguer des "micro-générations " (Pagis 2014). Les militants de la FEANF se construisent en opposition explicite à la génération formée dans les années 30 (Goebel 2016), celle des Senghor, des Houphouët-Boigny ou des Maga, dont les plus éminents représentants ont siégé à l'Assemblée de l'Union française, à l'Assemblée nationale, au Sénat, ont été Ministres ou secrétaires d'Etat d'un gouvernement français, celle dont les 
représentants sont aussi les premiers chefs d'Etat dans l'Afrique indépendante (Cooper 2014). Durant les années 50, c'est-à-dire avant les indépendances, la FEANF s'oppose à leurs compromis comme elle dénie toute pertinence aux théories de la négritude. Pour les militants de la fédération, séduits de plus en plus par un marxisme universaliste, la négritude est essentialiste et raciste. L'horizon d'attente est pour cette génération, non plus l'égalité avec les Français - mot d'ordre incontesté des grandes grèves qui secouent l'Afrique en amont et en aval du Code du travail d'Outremer de 1952, et mot d'ordre des étudiants restés à l'université alors régionale de Dakar, qui revendiquent un enseignement de même qualité que celui des Français - mais bien plutôt une Afrique libre, qui se construit pour et par elle-même. Et cela tant avant qu'après les indépendances. Cette Afrique libre, c'est aussi celle qu'appellent de leurs vœux les manifestants révolutionnaires des années 68, formés pour beaucoup d'entre eux au moule de la FEANF ou, pour les Malgaches, au moule de l'Association des Etudiants d'Origine Malgache (Rajaonah 2005-2006) ${ }^{41}$. C'est le cas en 1963 au Congo lors de la révolution dite des "Trois Glorieuses », où la jeunesse prendra le pouvoir, lors du « mai sénégalais » de 1968 ou lors du Mai malgache de 1972, où les manifestants réclament une " deuxième indépendance ", et remettent en cause les accords de coopération avec la France (Blum 2014) (Blum, Guidi, Rillon 2016). La FEANF anticipe déjà, dès les années 50, des ethos militants qui perdurent jusqu'au milieu des années 70 , que ce soit en France ou en Afrique. La venue en métropole joue ici un rôle de catalyseur qui permet de précéder une évolution un peu plus tardive en Afrique même. La FEANF accompagne le lent processus de dissolution de l'Empire français d'Afrique, que la seule date des Indépendances est loin de résumer. Les mouvements sociaux des années 60, tant en Afrique qu'en France peuvent aussi être lus à la lumière des décolonisations. Et ce, d'autant plus que les "pères de la nation " à qui l'on s'oppose dans les années 60 sont ceux-là même à qui s'opposaient déjà les militants de la FEANF dans les années 50.

Fig. A

Evolution disciplinaire des étudiants africains en France par rapport aux étudiants français

1963-1964 


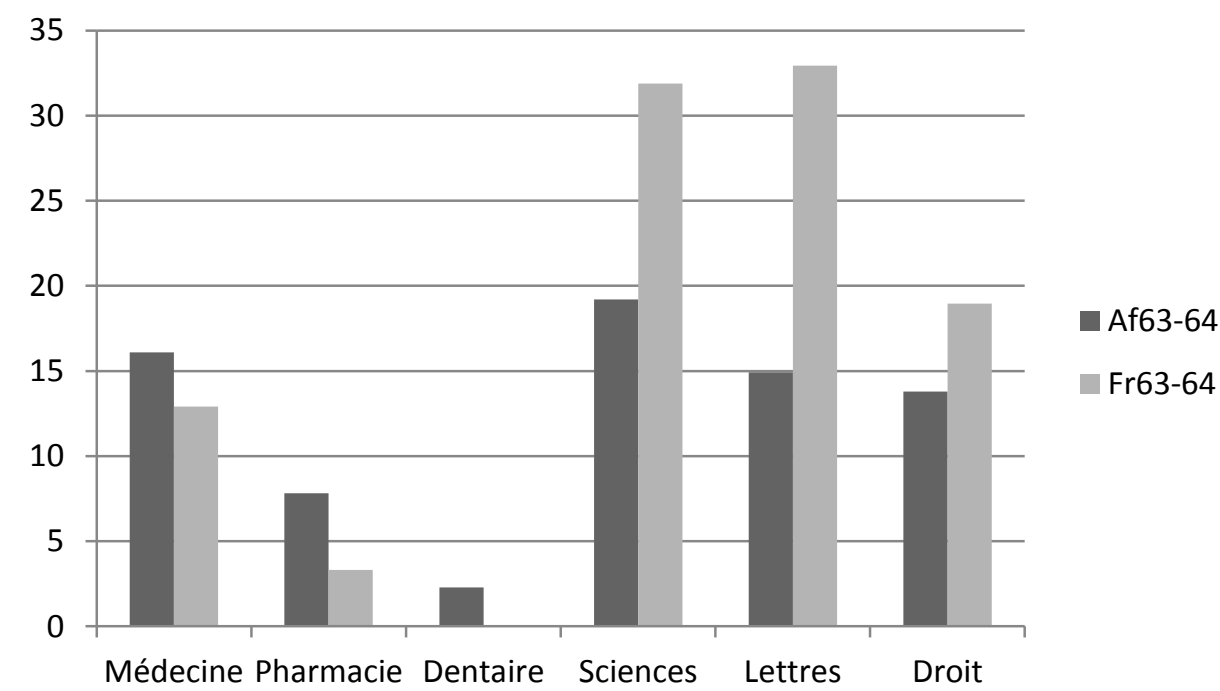

1974-1975

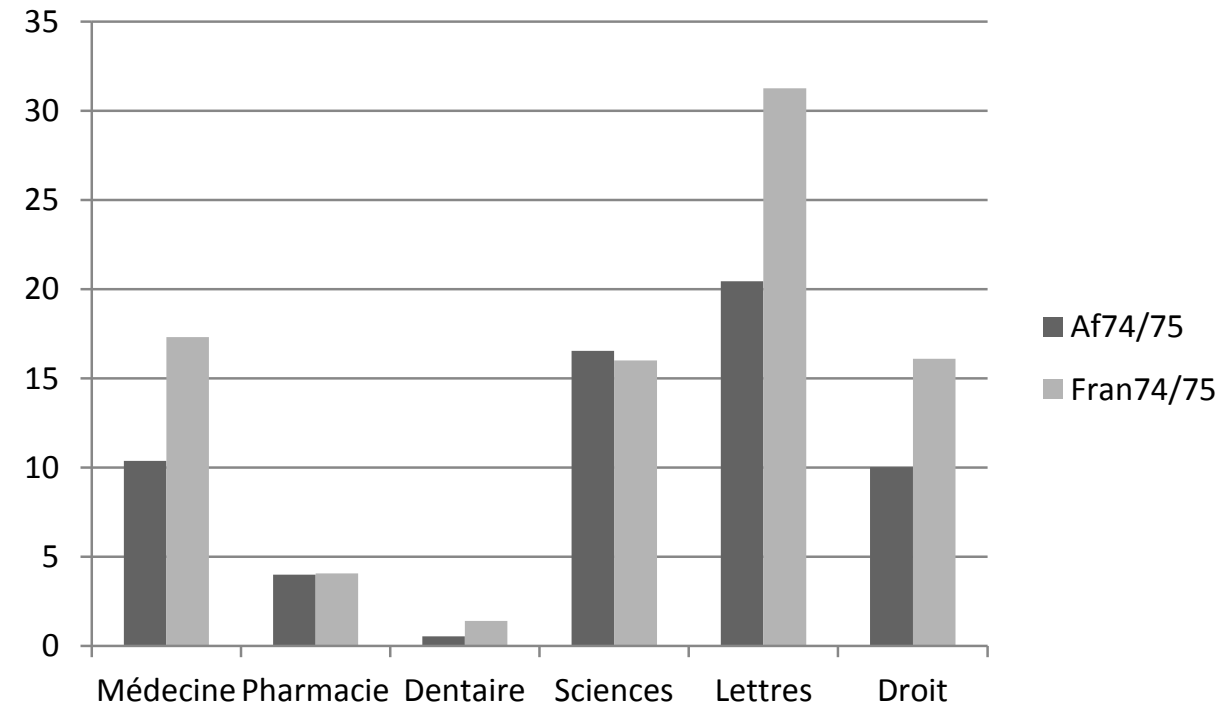

Fig B

Professions des pères des étudiants africains en France (AOF, AEF et Cameroun). 1959

\begin{tabular}{|l|l|l|l|l|l|l|l|l|}
\hline Fonctionnaires & Agriculteurs & Commerçants & $\begin{array}{l}\text { Chefs } \\
\text { notables }\end{array}$ & Employés & Magistrats & $\begin{array}{l}\text { Divers } \\
\text { diplomés }\end{array}$ & Divers & Prphelins \\
\hline $32 \%$ & $18 \%$ & $12 \%$ & $9 \%$ & $7,5 \%$ & $3 \%$ & $1 \%$ & $5,5 \%$ & $11 \%$ \\
\hline
\end{tabular}

Source : J. Bengono Ewondo, Tableau sociologique des étudiants d'Outre-Mer en France, Paris, École pratique des Hautes études, s.d., [1960]. - (Thèse sous la dir. de J. Vernant). 


\section{OUVRAGES CITES}

Amoussou, Bruno. 2009. L'Afrique est mon combat, Paris, L'Archipel.

BAZENGUISSA-GANGA, Rémy. 1997. Les voies du politique au Congo : essai de sociologie historique. Paris, Karthala.

BLUM, Françoise. 2014. Révolutions africaines : Congo, Sénégal, Madagascar. Presses universitaires de Rennes.

BLUM, Françoise. 2016. "Trajectoires militantes et (re)conversions : à propos de la FEANF », Habilitation à diriger des recherches, Paris, EHESS, 2016.

BLUM, Françoise. 2017. " De la Maison de la France d’Outre-mer à la résidence Lucien Paye ou comment un empire se défait » (à paraître).

BURRIN, Philippe. 1995. La France à I'heure allemande. Paris, Le Seuil.

CAMARA, Ousmane. 2010. Mémoires d'un juge africain. Dakar/Paris, Karthala/Crepos.

COOPER, Frederick. 2014. Citizenship between Empire and Nation. Princeton University Press.

Deltombe, Thomas, Domergue, Manuel, TAtSitsA, Jacob. 2011. Kamerun ! : Une guerre cachée aux origines de la Françafrique, 1948-1971. Paris, La Découverte.

DIANE, Charles. 1971. La FEANF et les grandes heures du mouvement syndical étudiant noir. Paris, Chaka.

DiENG, Amady Aly. 2009. Les grands combats de la FEANF. De Bandung aux indépendances. Paris, L'Harmattan.

DIENG, Amady Aly. 2003. Les premiers pas de la Fédération des étudiants d'Afrique noire en France. De l'union française à Bandung (1950-1955). Paris, L'Harmattan.

DIENG, Amady Aly. 2011. Mémoires d'un étudiant africain. Dakar, Codesria, 2 vol. volume I : de l'école régionale de Diourbel à l'université de Paris (1945-1960); volume II : de l'université de Paris à mon retour au Sénégal (1960-1967)

« Les Étudiants d’Outre-mer en France ». 1961. Sondages, n³.

GARBELY, Franck. 2005. L'assassinat de Félix Moumié : l'Afrique sous contrôle, documentaire, prod. Triluna film AG/Radio-télévion Suise/Arte/Aïe production SA, 53mn.

GOEBEL, Michael. 2016. Paris : capitale du Tiers-monde : comment est née la révolution anti-coloniale (19191939). Paris, La Découverte.

GUIMONT, Fabienne. 1985. Les Étudiants africains en France, 1950-1965. Paris, L'Harmattan.

GNALI, Aimée Mambou. 2001. Beto na beto : le poids de la tribu. Paris, Gallimard.

GUIMONT, Fabienne. 1985. Les Étudiants africains en France, 1950-1965. Paris, L'Harmattan.

KouroumA, Ahmadou . 1999. En attendant le vote des bêtes sauvages, Paris, le Grand livre du mois.

MANNHEIM, Karl. 2005. Le problème des générations; traduction de l'allemand par Gérard Mauger et Nia Perivolaropoulou ; introduction et postface de Gérard Mauger. Paris, A. Colin.

MAUGER, Gérard. 1994. " Gauchisme, contre culture et néo-libéralisme : pour une histoire de la 'génération de Mai 68' ", in CHEVALIER, Jacques (dir.). 1994. L'identité politique. PUF : 206-226.

MBEMBE, Achille. 1991. La naissance du maquis dans le Sud-Cameroun (1920-1960). Paris, Karthala.

N’DIAYE, Jean-Pierre. 1962. Enquête sur les étudiants noirs en France. Paris, Réalités africaines.

PAGIS, Julie. 2014. Mai 68 : un pavé dans leur histoire. Paris, Presses de Sciences Po. 
RAJAONAH, Faranirina. 2005-2006. , " Etre étudiant en métropole à l’avènement de l'indépendance :

l'Association des étudiants d'origine malgache de 1947 à 1960 », Afrika Zamani, nos. 13 \& 14, pp.1-22.

THIAM, Habib. 2001. Par devoir et par amitié, Paris, Editions du Rocher.

TRAORE, Sékou. 1985. La Fédération des étudiants d'Afrique noire en France. Paris, L'Harmattan.

$1 \quad$ Amady Aly Dieng est décédé à Dakar, le 13 mai 2015. Voir à son sujet : http://maitron-en-ligne.univparis1.fr/spip.php?article170559\&id_mot=9745

$2 \quad$ Outre les histoires déjà citées on peut consulter : Guimont, Fabienne. 1985. Les Étudiants africains en France, 1950-1965. Paris, L'Harmattan et Blum, Françoise. 2016. « Trajectoires militantes et (re)conversions : à propos de la FEANF », Habilitation à diriger des recherches, Paris, EHESS, 2016. Cet article est largement inspiré de cette HDR.

3 Des représentants de la FEANF siège à l'Office des étudiants d'Outre-mer (OEOM) mais ils sont évincés quand celui-ci devient Office de coopération et d'accueil universitaire (OCAU). Cela fait partie d'une reprise en main plus générale effectuée par les autorités françaises au détriment des Etats africains et de leurs étudiants.

$4 \quad$ Cette baisse, comme les fluctuations sont aussi visibles dans la fréquentation des congrès.

5 Dissoudre la FEANF avait été évoqué, dès le début des années 60, et à plusieurs reprises par les services du Ministère de l'intérieur. C'était alors le secrétariat des Affaires africaines de l'Elysée (les services de Jacques Foccart) qui s'y était opposé.

${ }^{6}$ Les catalogues des motions adoptées lors des divers congrès en font foi.

$7 \quad$ L'Etudiant d'Afrique noire eut un numéro spécial ( ${ }^{\circ} 23$, juin 1958) intitulé « Nazisme et Attila au Cameroun ». La fédération publia, sous l'égide de Jacques Vergès, une brochure Le Sang de Bandoeng (par Khar N’Dofene Diouf, E. Razafindralambo, Raymond Fardin et Jacques Vergès, Paris, Présence africaine, 1958, 63 p. qui comparait l'action française en Algérie aux crimes des nazis.

$8 \quad$ Archives nationales - Fonds Foccart - AG/5(F)/2610-SN/RG/10eme section/ $\mathrm{N}^{\circ} 178$.

$9 \quad$ Voir par exemple : Ministère de l'Intérieur, Service de coopération technique internationale de police. Juillet 1972. La Fédération des étudiants d'Afrique noire (FEANF), p.9-14.

10 Jean-Luc Godard, La Chinoise, Anouchka Films, 1967, 96mns.

11 Fonds Foccart - AG/5(F)/2610 - SN/RG/10eme section/N66 -La grève des étudiants africains.

12 Quand Deng Xiao Ping, en 1974, élabore la théorie des trois mondes, qui définit trois grands ensembles : les superpuissances, les pays développés, les pays en voie de développement et la Chine, cela provoque, comme chez les maoïstes français, de nouveaux clivages idéologiques, certains restant fidèles à la Chine et d'autres se tournant vers l'Albanie.

$13 \quad$ Les archives de la section coloniale du PCF ont disparu. 
14 Cette aide financière est attestée à plusieurs reprises par les rapports des renseignements généraux et Ousmane Camara (Camara $2010: 45$ ).

15 AN-CAC (Archives nationales- Centre des archives contemporaines) -- Dossier 960134/18 - Ministère de l'Intérieur. 1966. Les Étudiants d'Afrique noire, évolution générale en 1966, p.17-18.

16 Le 21 février est traditionnellement une journée anti-coloniale, à l'origine instituée en mémoire de grèves en Egypte et réactivée durant la guerre d'Algérie

17 Fonds Foccart - AG/5(F) 2624.

18 Entretien avec Makan Keita, Paris, décembre 2014.

19 AN-CAC- Dossier 960134/18. Le dossier comprend le texte de la pièce.

$20 \quad$ Entretien avec Joseph Van Den Reysen, Toulouse, juin 2015.

21 Entretiens avec François Codjo-Azodogbehou, Cotonou, juillet 2015.

22 Fonds Foccart - AG/5(F)/2610-SN/RG/10eme section/Nº 178.

$23 \quad$ Ibid. - Note du 20 Octobre 1964

24 Entretien avec Robert Dossou et Boniface Edey-Gamassou, Paris, décembre 2014 - Entretien avec Valentine Quenum, Cotonou, juillet 2015.

25 Sur l'OEOM-OCAU voir Guimont, ibid.

26 Les éléments préparatoires de l'enquête publiée dans Sondages sont réunis dans AN-OCAU 19780596/51 - Sous-dossier « Enquête auprès des étudiants O.M. en France.

$27 \quad$ Il s'agit d'un processus et il est donc illusoire de chercher des dates précises. L'occupation de postes de pouvoir par les étudiants de la FEANF contribuent à cette redéfinition des élites et à leur reproduction via le système scolaire " à la française ".

28 Par exemple, en Afrique de l'Ouest la place réciproque sur l'échiquier scolaire des castés ou non castés.

29 Entretien avec Bruno Amoussou, Cotonou, juillet 2015.

30 Ces lettres m'ont été communiquées par Constantin Katsakioris que je remercie.

$31 \quad$ Fonds Kaldor - 503-J4- Rome le 19 octobre 1971, Michel Ndoh à son excellence Monsieur G. Pompidou.

32 Les quatre Communes, Saint-Louis, Dakar, Rufisque et Gorée avaient un statut particulier. Leurs ressortissants étaient citoyens français depuis 1916.

33 Ce qui suit provient d'un entretien réalisé avec Boubakar Ly, Dakar, Janvier 2015. Pour une biographie plus complète voir la notice réalisée par Françoise Blum: http://maitron-en-ligne.univparis1.fr/spip.php?article170686\&id_mot=9745.

$34 \quad$ Ce qui suit provient d'un entretien avec Gédéon Dassoundo, Cotonou, juillet 2015.

$35 \quad$ Prononcé par Mathieu Kérékou le 30 novembre 1972.

$36 \quad$ lbid.

37 Entretien avec Valentine Quenum, Cotonou, juillet 2015.

Henri Lopes, écrivain, plusieurs fois ministre puis ambassadeur à Paris en fin de carrière.

$39 \quad$ Entretien avec Boubakar Ly, Dakar, janvier 2015.

40 Mamadou Dia, président du conseil, fut mis en accusation et jugé pour tentative de coup d'état, en 1962. 